This document is the unedited Author's version of a Submitted Work that was subsequently accepted for publication in nano Letters, copyright (c) American Chemical Society after peer review. To access the final edited and published work see https://pubs.acs.org/doi/abs/10.1021/acs.nanolett.9b04808, see http://pubs.acs.org/page/policy/articlesonrequest/index.html.

\title{
Phase selection in self-catalyzed GaAs nanowires
}

Federico Panciera ${ }^{1,2, *}$, Zhaslan Baraissov ${ }^{2,3}$, Gilles Patriarche ${ }^{1}$, Vladimir G. Dubrovskii ${ }^{4}$, Frank Glas $^{1}$, Laurent Travers ${ }^{1}$,Utkur Mirsaidov ${ }^{2,3}, *$, Jean-Christophe Harmand ${ }^{1}$

${ }^{1}$ Université Paris-Saclay, CNRS, Centre de Nanosciences et de Nanotechnologies, 91120, Palaiseau, France.

${ }^{2}$ Centre for BioImaging Sciences, Department of Biological Sciences, National University of Singapore, 14 Science Drive 4, 117557, Singapore

${ }^{3}$ Centre for Advanced 2D Materials and Department of Physics, National University of Singapore, Science Drive 4, 117543, Singapore

${ }^{4}$ ITMO University, Kronverkskiy pr. 49, 197101 St. Petersburg, Russia

Keywords: nanowires, in situ TEM, crystal growth, polytypism, crystal phase

Crystal phase switching between the zincblende and wurtzite structures in III-V nanowires is crucial from the fundamental viewpoint as well as for electronic and photonic applications of crystal phase heterostructures. Here, the results of in situ monitoring of self-catalyzed vapor-liquid-solid growth of GaAs nanowires by molecular beam epitaxy inside a transmission electron microscope is presented. It is demonstrated that the occurrence of the zincblende or wurtzite phase in self-catalyzed nanowires is determined by the sole parameter, the droplet contact angle, which can be finely tuned 
by changing the group III and V fluxes. The zincblende phase forms at small $\left(<100^{\circ}\right)$ and large $(>$ $125^{\circ}$ ) contact angles, while pure wurtzite phase is observed for intermediate contact angles. Wurtzite nanowires are restricted by vertical sidewalls, while zincblende nanowires taper or develop the truncated edge at their top. These findings are explained within a dedicated model for surface energetics. These results give a clear route for the crystal phase control in Au-free III-V nanowires. On a more general note, in situ growth monitoring with atomic resolution and at the technologicalrelevant growth rates is shown to be a powerful tool for the fine-tuning of material properties at the nanoscale.

Growth of III-V semiconductor nanowires (NWs) using the vapor-liquid-solid (VLS) method can result in the crystal structures different from their bulk phase. ${ }^{1,2,3}$ In GaAs NWs, for example, stable zincblende (ZB) phase coexists with metastable wurtzite (WZ) structure. ${ }^{4}$ Due to a relatively small difference in cohesive energy of the two phases, a modest change in the growth conditions is sufficient to switch from one phase to the other. The formation probabilities of the two phases are often close to each other, resulting in NWs having a mixed-phase structure and stacking faults. Controlling the crystal phase purity is critical for technological applications, because different crystal structures of the same chemical compound present different electronic, photonic or phononic properties, with discontinuities at their common interfaces. At the same time, controlled switching between the two phases enables synthesis of novel heterostructures with different electronic and optoelectronic properties in each phase. ${ }^{5,6}$ Remarkably, the valence and conduction bands of the two phases are misaligned, so that small sections of one phase within the other effectively confine charge carriers. In contrast to compositional heterojunctions, crystal phase heterostructures have intrinsically abrupt interfaces and hence do not suffer from the alloy intermixing at the interface. This feature has been proven crucial for the fabrication of crystalphase quantum dots with exceptional properties. ${ }^{7,8,9,10}$ 
Despite the compelling need for phase control, the lack of fundamental understanding of the governing mechanisms prevents growing these nanostructures reliably. Several models have been proposed to date to explain the crystal phase switching. Even though the suggested mechanisms differ, there is a general consensus that the phase selection occurs at the moment of nucleation of each new monolayer (ML). ${ }^{11}$ It has also been highlighted that the key parameter that determines the choice of the phase is the contact angle between the droplet and the crystal (hereafter referred to as "the contact angle"). ${ }^{11,12,13}$ A change in the contact angle alters the balance of capillary forces at the triple-phase line (TPL). It may thus render the nucleation site more favorable for one phase with respect to the other. Unfortunately, despite the evident importance of controlling the contact angle, this parameter cannot be monitored or directly adjusted during growth in a standard reactor. Only the experiments conducted by in situ transmission electron microscopy (TEM) ${ }^{12,14,15}$ helped to shed light on the relationship between the growth parameters and the contact angle and provided valuable information for developing the droplet engineering strategies. However, these experiments treated Au-catalysed nanowires, not desirable for integration with silicon platform, and grown at rates much lower than in the real epitaxy systems.

Here, we present direct observations of the growth of self-catalyzed GaAs nanowires by molecular beam epitaxy (MBE) using in situ TEM. We chose to study self-catalyzed III-V NWs, as opposed to Au-catalysed $\mathrm{NWs}^{12}$ due to their higher technological relevance and because this growth mode gives access to the full range of contact angles. Indeed, the droplet size can be continuously decreased until total consumption by supplying an excess of group $\mathrm{V}$ element. This property allows us to monitor the change of crystal phase over a wide range of contact angles and discover a mechanism for the phase transition that occurs at small angles. We show that multiple 
mechanisms are involved in the phase selection and develop a theoretical model to explain the observed behavior.

GaAs NWs were grown using specially designed MBE sources directly fitted on the microscope. ${ }^{15}$ The material fluxes were adjusted to obtain an average growth rate on the order of $1 \mathrm{ML} / \mathrm{s}$, which is the typical MBE growth speed in standard systems (see Methods). By changing the ratio of Ga and As fluxes, we deliberately modified the size of the catalyst droplet and hence the contact angle. Starting from a large droplet and gradually decreasing the droplet size, the first transition from $\mathrm{ZB}$ to $\mathrm{WZ}$ is observed at a contact angle of $\sim 125^{\circ}$. Further decrease of the droplet size leads to the second transition, from $\mathrm{WZ}$ to $\mathrm{ZB}$, at a contact angle of $\sim 100^{\circ}$.

We systematically observe three distinct regimes that are characterized by different morphologies and the formation of a specific phase. Large contact angles result in the ZB phase, where the formation of each new ML is quasi-instantaneous and the edge truncation is present at the TPL (Figure 1a). At moderate contact angles below $125^{\circ}$, the $\mathrm{WZ}$ phase forms and the edge truncation is absent. The ML nucleates at the TPL and extends across the NW/droplet interface through a slow step flow (Figure $1 \mathrm{~b}$ ). For small contact angles below $\sim 100^{\circ}$, the ZB phase forms again. This second phase transition was predicted earlier to occur at contact angles well below $90^{\circ},{ }^{12}$ but this was never confirmed experimentally. Remarkably, our observation reveals an important similarity between the growths of GaAs NWs at moderate (between $100^{\circ}$ and $125^{\circ}$ ) and small $\left(<100^{\circ}\right)$ contact angles. In both cases, no edge truncation is observed in NWs, the ML nucleation occurs at the TPL, and lateral spreading of the ML is slow (Figure 1c). The two latter features are observed independently of the crystal phase, and hence must be entirely related to the morphology of the TPL. In our conditions, the amount of As from the liquid phase is not sufficient to form the entire ML, ${ }^{15}$ thus the missing As must be supplied either from vapor or solid phase. If 
As is supplied from vapour, the growth duration of the full ML is limited by the As refill from vapor, which is relatively slow. This must be the case for moderate and small contact angles, where no edge truncation is present. On the other hand, if As is transferred directly from the truncated facet (whose size oscillates within the ML growth cycle), the ML can be completed quasiinstantaneously. This situation corresponds to large contact angles above $125^{\circ}$, where the edge truncation serves as an extra source of material for the rapid completion of the whole ML.

a
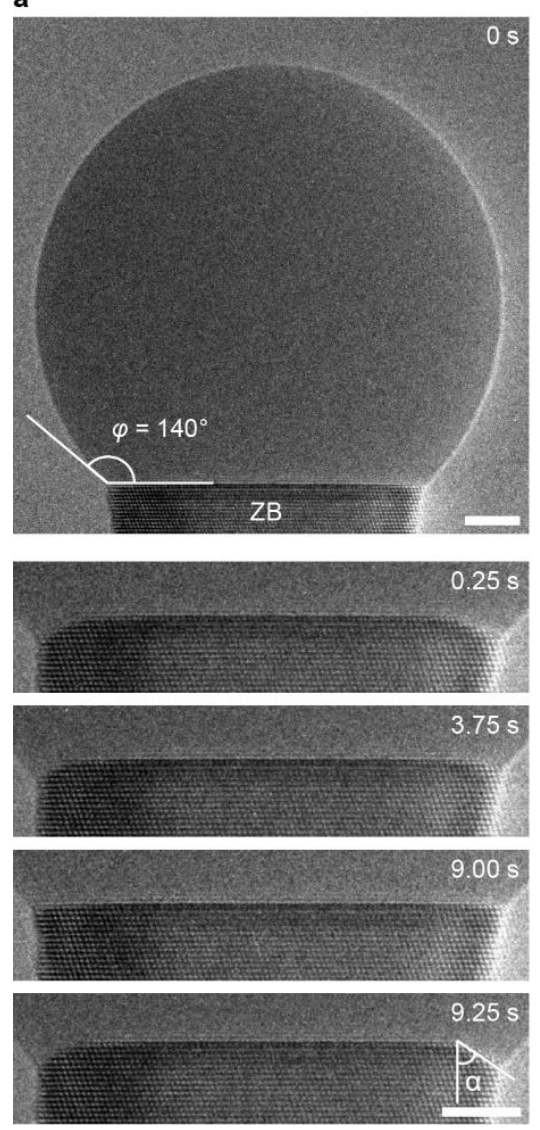

b
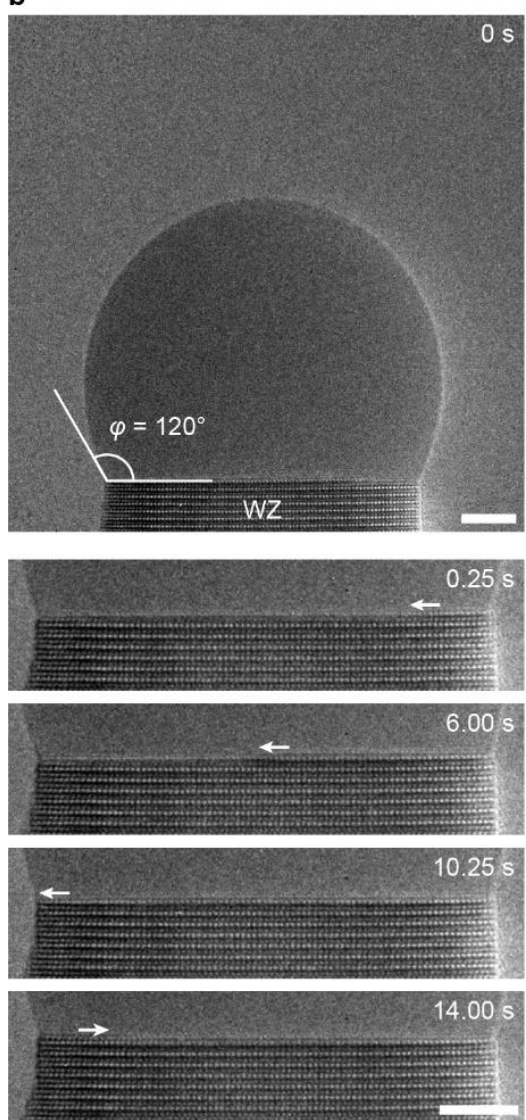

c
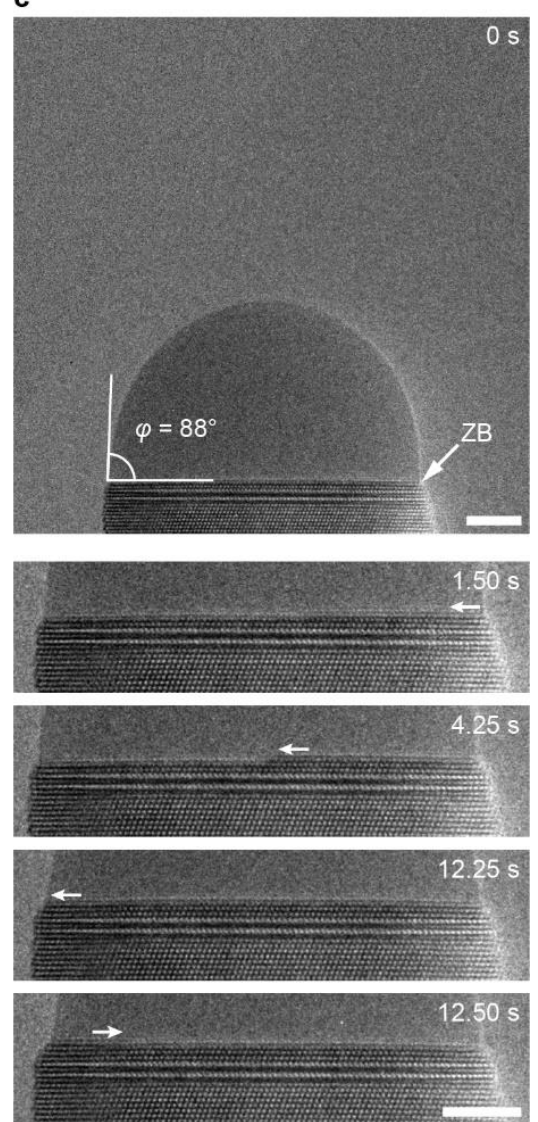

Figure 1. Growth mode and phase selection at different contact angles. All images are recorded from the same self-catalysed GaAs NW having a diameter of $\sim 30 \mathrm{~nm}$ and growing at $420{ }^{\circ} \mathrm{C}$ under different fluxes of As and Ga. The As/Ga flux ratio was changed during growth to tune the volume of the catalyst droplet and consequently the contact angle. Scale bars are $5 \mathrm{~nm}$ and the ML numbers refer to Figure 2 a. a) Images extracted from Movie 1 recorded during growth of ZB NW section (with an As flux of $0.06 \mathrm{~nm} / \mathrm{s}$ and a Ga flux of $0.15 \mathrm{~nm} / \mathrm{s}$ ). Each new ML grows quasi-instantaneously (in one frame) and its formation corresponds to the development of the edge truncation. The truncated facet is inclined at the angle $\alpha$ with respect to the vertical and is wetted by the liquid catalyst. The amount of truncation oscillates with the period of ML growth, it decreases slowly but then jumps back to its maximum size with the next ML nucleation. Note that the truncation appears simultaneously on both sides of the NW; asymmetric truncations are observed in the presence of stacking faults or near the transition from ZB to WZ. The two nucleation 
events correspond to MLs \#17 and \#18. b) Images extracted from Movie 2 (MLs \#226 and \#227) recorded during growth of WZ NW section (with an As flux of $0.08 \mathrm{~nm} / \mathrm{s}$ and a Ga flux of $0.15 \mathrm{~nm} / \mathrm{s}$ ). The step flows slowly across the planar top facet of the NW; the position of the step is indicated by the arrows. c) Images extracted from Movie 3 (MLs \#377 and \#378) recorded during growth of ZB NW section (with an As flux of $0.8 \mathrm{~nm} / \mathrm{s}$ and Ga shutter closed). Similar to b), the growth occurs by a slow step flow and the edge corner is sharp. The two nucleation events correspond to MLs \#377 and \#378.

To understand the mechanisms for switching between these three regimes, we performed experiments in which the droplet volume was changed several times by modulating either Ga or As flux [see the Supporting Information (SI) 1 and 2 for more details]. The droplet volume can change by one of the two possible mechanisms. The first one is the change in NW diameter, where the system tends to maintain a constant contact angle. In this case, unbalanced capillary forces at the TPL lead to a change of the NW top diameter through the introduction of the inclined side facets. ${ }^{16}$ Hence, the NW adjusts its diameter to keep the stable contact angle. ${ }^{17,18,19}$ The second one is changing the contact angle of the droplet at a constant NW diameter, leading to the phase transition when the contact angle reaches a specific critical value. Figure $2 \mathrm{a}$ shows that the phase transition occurs at the two critical angles, $\varphi_{\min }=100^{\circ}$ and $\varphi_{\max }=125^{\circ}$, regardless of whether the droplet volume is increased or decreased. Between the two critical angles, the crystal phase is WZ, switching to ZB outside this range.

This central result cannot be explained within the existing models of polytypism. ${ }^{11,12}$ Indeed, one central conclusion of both models is the maximum probability for the $\mathrm{WZ}$ phase formation at $\varphi=90^{\circ}$, which contradicts our in situ data. It should be noted that both models ignore possible tapering of the NWs, which modifies the surface energy balance at the TPL. 


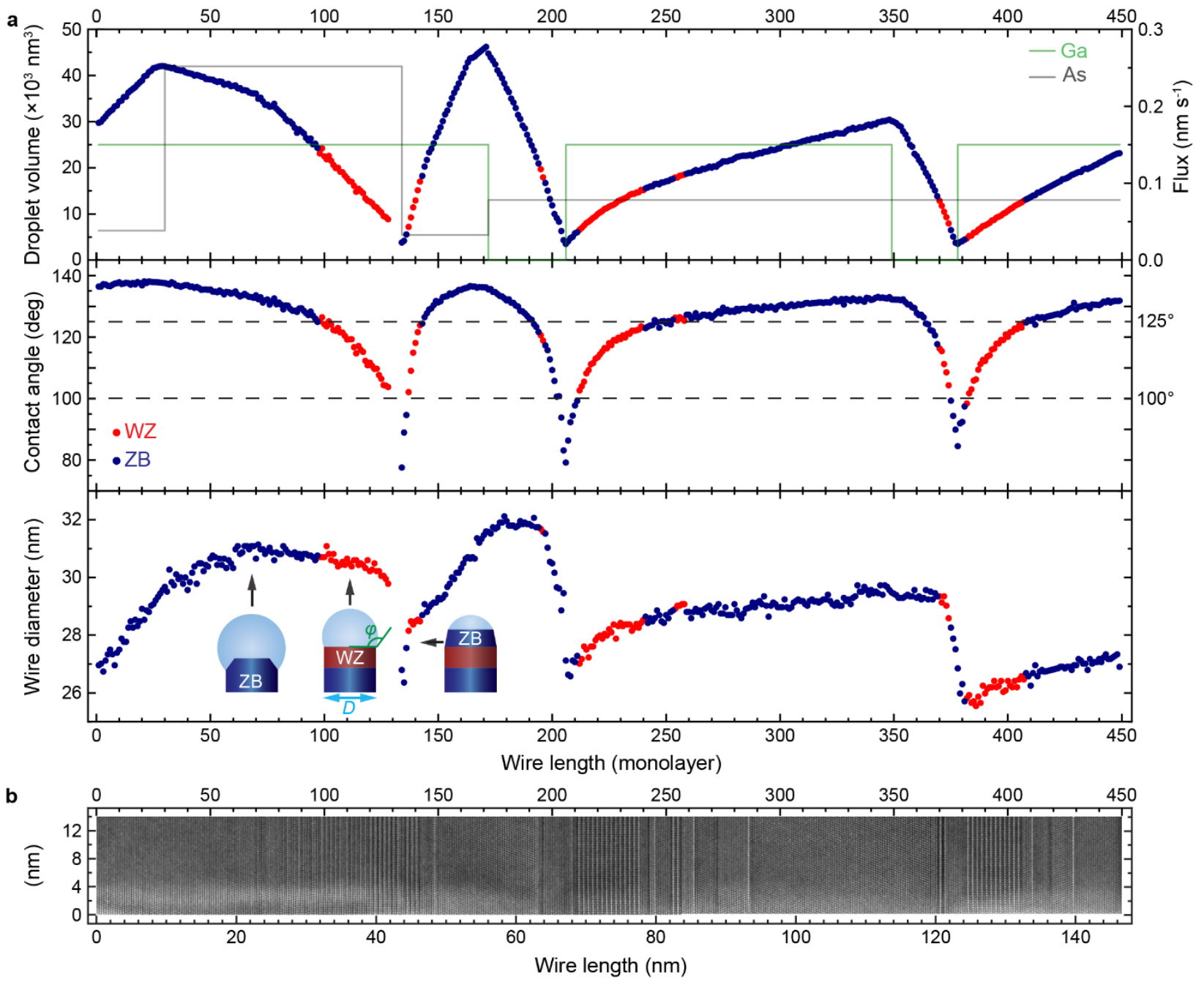

Figure 2. Time evolution of the NW morphology under varying As and Ga fluxes. a) The droplet volume (top), contact angle (middle) and base diameter (bottom) are plotted as functions of the NW length. b) Image of a slice of the NW obtained by composing several TEM images. Each data point is measured on the image in which the ML nucleation is observed and color-coded for the two crystal phases (blue for ZB and red for WZ). The effective fluxes of As and Ga are calculated from the axial growth rate of the NW and the change in the droplet volume (see SI 2). Data points corresponding to ML \# 129-133 were not recorded.

We now consider in more detail the NW morphology to fully understand the phase switching mechanisms. Figure 2 shows that the two phases respond to the change in the droplet volume in very different ways. WZ NW maintains vertical sidewalls during a significant change in the contact angle (from $\varphi_{\min }=100^{\circ}$ to $\varphi_{\max }=125^{\circ}$ ). A slight increase in the top diameter visible in some cases is due to radial growth through the vertical step flow (see Movie 4), which occurs later than the axial growth and does not affect the crystal phase selection. Conversely, the diameter of ZB 
NW rapidly adjusts to the changing droplet volume by outward tapering (taper angle $\theta<0$ ) for large or inward tapering $(\theta>0)$ for small contact angles. It is noteworthy that the phase transition from WZ to ZB occurs quasi-simultaneously with tapering of the NW. A natural question to ask is whether the phase change triggers the NW tapering or vice versa. In all our experiments, we observed that the phase transition occurs at the same time or slightly after developing of an inclined side facet in the WZ segment, as shown Figure 3 ( see also Figure S9 and Movie 4). After the phase switches to ZB, tapering proceeds by developing well defined $\{110\}$ facets (see Figure 3 and Figure S9). At small contact angles, the phase change is thus promoted by tapering of the NW sidewalls.
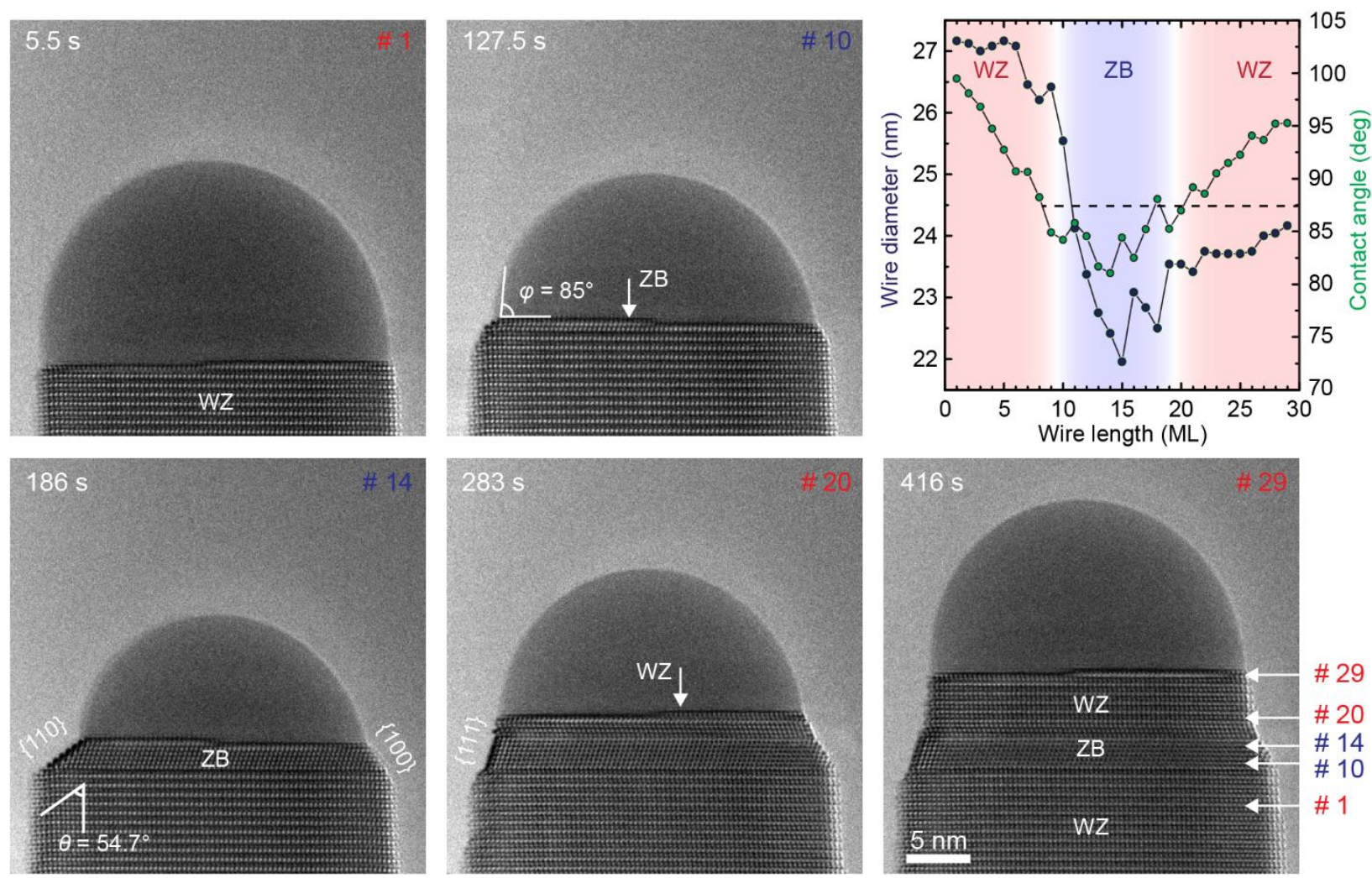

Figure 3. Sequence of images showing the phase switching caused by the NW tapering at small contact angles. Each image was captured when the ML was half-grown to make its crystal phase clearly identifiable. The graphs show the NW diameter and the contact angle versus the NW length expressed in MLs. The initial conditions are: substrate temperature $=480{ }^{\circ} \mathrm{C}$, As pressure $=1.2 \times 10^{-5} \mathrm{mbar}$, Ga shutter closed. After ML \#14, the Ga source was opened and kept at a temperature of $940^{\circ} \mathrm{C}$. When the contact angle decreases, tapering starts within the WZ phase at ML \#10. When the contact angle increases, the crystal phase switches to WZ at ML \#20 and simultaneously the NW sidewalls 
become vertical. The diameter increase after ML \#14 is not caused by an inverse tapering at the interface but is rather due to radial growth, which reorganizes the $\{110\}$ facets into the vertical $\{111\}$ facets.

This mechanism has some features in common with the phase change at large contact angles, which, according to Reference 12, is determined by developing a truncated facet at the TPL. Even though the phase switching at the two critical contact angles is governed by different mechanisms (either tapering or truncation), a unifying pattern emerges. Indeed, in both cases the phase switching from $\mathrm{WZ}$ to $\mathrm{ZB}$ is accompanied by a modification of the growth front. At the large critical angle, the side facet at the NW top changes from vertical to truncated, with the truncation inside the liquid phase, while at the small critical angle the side facet changes from vertical to tapered, not wetted by the liquid phase. The development of these novel inclined facets changes the surface energy balance at the TPL, which in turn determines the preferred crystal phase. The ZB phase always emerges in presence of an inclined facet, suggesting that the edge line between the inclined facet and the top <111> facet represents the preferential nucleation site for ZB NWs. Based on these observations, we develop a unifying model that predicts the morphology of the growth interface and the preferred crystal phase as a function the contact angle $\varphi$.

Generalizing the methods developed by Tersoff ${ }^{17,12}$ and Dubrovskii, ${ }^{19}$ we derive the two equations that represent the difference of surface energy between a given morphology, which has either tapered (t) sidewalls or truncated (tr) edges, and the reference state having vertical sidewalls and $90^{\circ}$ edges at the TPL. In addition to the previous models, we take into account that the NW can adopt either ZB or WZ structure at the growth front and hence the solid-vapor surface energies are phase-dependent ( $k=\mathrm{ZB}$ or WZ). These equations are given by

$$
\begin{aligned}
& \Delta F_{t}^{k}(\theta, \varphi)=\frac{\gamma_{\theta V}^{\kappa}}{\cos \theta}-\gamma_{0 V}^{\kappa}-\left(\gamma_{S L}+\gamma_{L V} \cos \varphi\right) \tan \theta \\
& \Delta F_{t r}^{k}(\alpha, \varphi)=\frac{\gamma_{\alpha L}}{\cos \alpha}-\gamma_{0 V}^{\kappa}-\gamma_{S L} \tan \alpha+\gamma_{L V} \sin \varphi
\end{aligned}
$$


Here, $\gamma_{S L}$ and $\gamma_{L V}$ are the phase-independent surface energies of the horizontal solid-liquid and liquid-vapour interfaces; $\gamma_{\alpha L}$ is the solid-liquid surface energy of a ZB truncated facet, inclined at the angle $\alpha$ to the vertical; $\gamma_{0 V}^{Z B}$ is the surface energy of vertical $\{110\}$ sidewall facet of ZB NW, which represents the reference; $\gamma_{0 V}^{W Z}$ is the surface energy of vertical $(1 \overline{1} 00)$ sidewall facet of WZ $\mathrm{NW}$, which is lower than $\gamma_{0 V}^{Z B}$; and $\gamma_{\theta V}^{\kappa}$ is the surface energy of a tapered facet, inclined at the angle $\theta$ to the vertical. Positive or negative angles $\theta$ correspond to narrowing or widening facets, leading to the NW tapering or inverse tapering, respectively. To model the NW morphology and phase versus the contact angle $\varphi$, we use a single set of surface energies for Ga-GaAs system gathered from the literature, ${ }^{19,20,21,22,23}$ the measured angles of the inclined facets $(\theta$ and $\alpha$ ), and the knowledge on the phase trends deduced from our experimental observations (see Methods). By doing so, the equations contain two unknowns, the solid-liquid surface energies $\gamma_{S L}$ and $\gamma_{\alpha L}$.

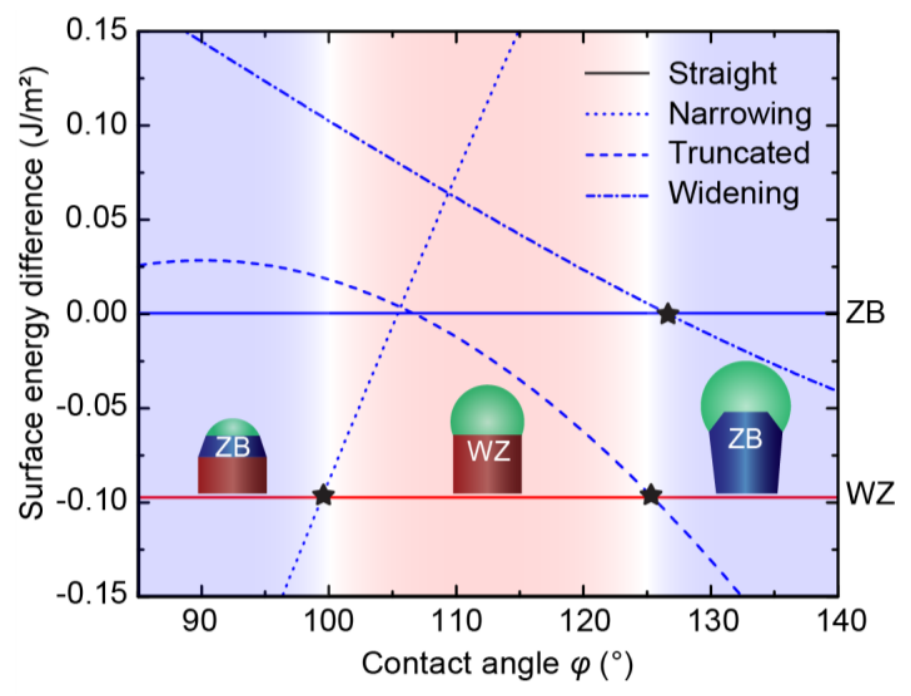

Figure 4. Model for the NW morphology and phase selection, showing the transitions from tapered ZB NWs at small contact angles to vertical WZ NWs at intermediate contact angles and back to ZB NWs with wetted truncated edges at large contact angles. Blue zero-level line corresponds to vertical (110) ZB facets and the red horizontal line to vertical (1100) WZ facets. The increasing curve for the narrowing (110) ZB facet intercepts with the WZ one at $\varphi_{\min }=100^{\circ}$, corresponding to the preferred ZB structure below $100^{\circ}$. The curve for the wetted truncated facet crosses with the $\mathrm{WZ}$ one at $\varphi_{\max }=125^{\circ}$, showing that the crystal phase is ZB above $\varphi_{\max }$. The morphology of these ZB NWs is first vertical, transitioning to inverse tapered when the contact angle further increases. In both cases, the growth front is truncated. The region between the two critical angles on the WZ line corresponds to vertical WZ NWs. 
The curves obtained from equation 1 and 2 are plotted by using the known surface energy values reported in Table 1 and by adjusting the two unknown values $\left(\gamma_{S L}\right.$ and and $\left.\gamma_{\alpha L}\right)$ in order to fit the two critical contact angles corresponding to the ZB-WZ phase transitions. With this method, we are able to deduce the plausible values of $\gamma_{S L}=0.593 \mathrm{~J} / \mathrm{m}^{2}$ and $\gamma_{\alpha L}=0.566 \mathrm{~J} / \mathrm{m}^{2}$, which appear very close to each other (more details are given in SI, paragraph S4). Overall, the model explains very well our experimental observations and agrees with the earlier results. It is seen that the vertical WZ configuration is preferred for intermediate contact angles from $\varphi_{\min }=100^{\circ}$ to $\varphi_{\max }=125^{\circ}$, while for smaller and larger angles, the tapered and truncated ZB configurations are more favorable. Above $125^{\circ}$, the growth front is truncated and the formation of WZ phase is prevented. There is a narrow range of contact angles (from $125^{\circ}$ to $127^{\circ}$ ) where $\mathrm{ZB} \mathrm{NWs} \mathrm{have}$ vertical $\{110\}$ sidewalls, while for larger $\varphi$ ZB NWs exhibit inverse tapering, as often seen in selfcatalyzed GaAs NWs ${ }^{23,24}$. Our model also contemplates a possible formation of a truncation at very small contact angles (smaller than $55^{\circ}$, not shown in Figure 4), as predicted earlier in Reference 12. However, at contact angles smaller than $\varphi_{\min }$, development of the tapered nonwetted facet is more favorable and hence the truncation is suppressed. Furthermore, our experiments show that when the contact angle approaches $55^{\circ}$, the droplet unpins from the TPL and moves freely on the top (111) surface (Figure S9). We found, however, that the minimum angles $\varphi_{\min }$ for the WZ-to-ZB phase switching vary in a range between $85^{\circ}$ and $100^{\circ}$. We attribute this variability to different sidewall structures of the NWs (see SI, paragraph S3).

Precise control of the morphology of the $\mathrm{ZB}$ and $\mathrm{WZ}$ segments in crystal phase heterostructures is crucial for various applications. In this context, the phase change at large angles has two main advantages. First, it is more predictable since, for all the investigated growth conditions, it occurs in a very narrow range of angles around $125^{\circ}$ (Figure S6). Second, it does not 
induce any significant change in the NW diameter, particularly if the maximum contact angle is smaller than $127^{\circ}$. Nevertheless, the phase change at small angles cannot be ignored as it occurs almost inevitably at the two crucial steps of NW growth. This transition should be observed at the beginning of growth ${ }^{11}$, because the droplet has a much smaller contact angle when sitting on the planar substrate surface than on top of developed NWs. It also occurs at the end of growth, when the contact angle decreases when the droplet is completely ${ }^{25}$ or partially ${ }^{26}$ consumed. Furthermore, the formation of quantum-thin GaAs NWs requires a stage of droplet shrinking under high As fluxes, where the contact angle is small. ${ }^{27}$

It is worth noticing that when the droplet volume decreases rapidly, the WZ insertion between the two ZB segments becomes very short and virtually disappears in the most extreme case (see Figure 2a, ML \#200). This provides a clear route for the fabrication of pure ZB GaAs NWs, avoiding the phase mixing at the NW top. Previous studies ${ }^{24}$ suggested that self-catalyzed GaAs NWs should be almost exclusively ZB due to a low surface energy of liquid Ga compared to Au. Our results show, however, that the WZ phase in such NWs is easily achievable under relatively high V/III ratios. Predominantly ZB phase observed previously is explained by effectively Ga-rich growth conditions employed in these works. Ga-rich environment guarantees that the Ga droplet is not consumed by an excessive As flux. On the other hand, it leads to droplet inflation, truncated growth interface and inverse tapering, following the described growth scenario at large contact angles.

In conclusion, our in situ growth monitoring and modeling of self-catalyzed GaAs and $\mathrm{GaSb}$ (see S9) NWs clearly shows the crystal phase switching depending on the sole parameter, the droplet contact angle, which can easily be regulated by the incoming material fluxes. The crystal phase can be changed in a regular way from pure ZB to pure WZ with the well-controlled lengths 
of crystal phase segments, which gives a clear route for obtaining crystal phase heterostructures in the Au-free approach. The method can be extended to other III-V NWs and demonstrates the capability of in situ method for delicate manipulations with the morphology, crystal phase and ultimately physical properties of nanomaterials.

\section{Methods}

\section{Growth}

We observed the growth of GaAs NWs in situ using a Cs corrected Titan environmental TEM (ETEM), equipped with custom-made MBE sources. These sources are highly collimated so that the evaporated material only deposits onto the sample. The substrate was a Protochips heating $\mathrm{SiC}$ membrane with holes of $10 \mu \mathrm{m}$ diameter. Pure Ga and As were loaded into boron nitride crucible and heated to about 930 and $320^{\circ} \mathrm{C}$, respectively, to obtain a volumetric flux on the order of 0.3 $\left(\mathrm{nm}^{3} / \mathrm{s}\right)$ per $\mathrm{nm}^{2}$, which corresponds to a growth rate of about 1 ML per second (see SI, paragraph S2). This growth rate was selected as a trade-off between realistic growth conditions for a standard reactor and a growth speed that allows recording high-quality data. By keeping the source temperature constant, it was possible to adjust the flux of As through a needle valve and consequently control the growth rate. Ga was initially deposited on the substrate at a temperature of $500{ }^{\circ} \mathrm{C}$ and when the $\mathrm{Ga}$ droplets reached the size of 20-30 nm, Ga was introduced and temperature decreased to $420{ }^{\circ} \mathrm{C}$ to promote the growth of NWs. Due to the polycrystalline nature of the $\mathrm{SiC}$ substrate, NWs started growing in arbitrary directions on the substrate or freely suspended in vacuum in correspondence to the holes. The sample was then tilted to orient the selected NW on a specific zone axis, generally $\langle 110\rangle$. Typical experiments were carried out at a growth rate between 0.05 and $1 \mathrm{ML} / \mathrm{s}$ and high-resolution movies were recorded using a Gatan US1000 camera at a rate of 4 frames per second. Images were analyzed using an automated script that determines the relevant parameters such as the NW diameter, the volume of catalyst droplet and its contact angle (see SI S1).

\section{Model}

Equations 1 and 2 are derived from theoretical work ${ }^{19}$ and plotted in Figure 4 using the surface energy values reported in Table 1 . Here, thanks to the experimental determination of the critical 
contact angles corresponding to the crystal phase switching and identification of different facets, we can assign the accurate values for the surface energies. For the angles $\theta$, we distinguish between narrowing (n), vertical (v) and widening (w) facets. The angle $\theta(n)$ and the truncation angle $\alpha$ are directly measured form the TEM images. For widening of ZB GaAs NWs, we deduced that the inverse tapering proceeds by an alternation of vertical (110) and outward tapered (111)B sidewall facets. Therefore, in the model, we assign $\theta(w)$ and the corresponding surface energy value to the (111)B facet.

Table 1: Parameter values used to plot Figure 4 based on Eqs. (1) and (2).

\begin{tabular}{|c|c|c|c|c|c|c|c|c|c|c|c|}
\hline $\begin{array}{c}\text { Model } \\
\text { Para- } \\
\text { meters }\end{array}$ & $\begin{array}{c}\gamma_{\theta V}^{Z B}(\mathrm{w}) \\
=\end{array}$ & $\begin{array}{c}\gamma_{\theta V}^{Z B}(\mathrm{n}) \\
=\end{array}$ & $\begin{array}{c}\gamma_{\theta V}^{W Z}(\mathrm{v}) \\
=\end{array}$ & $\begin{array}{c}\gamma_{L V} \\
=\end{array}$ & $\begin{array}{c}\varphi_{\min } \\
(\mathrm{deg})\end{array}$ & $\begin{array}{c}\varphi_{\max } \\
(\mathrm{deg})\end{array}$ & $\begin{array}{c}\theta(\mathrm{w}) \\
(\mathrm{deg})\end{array}$ & $\begin{array}{c}\theta(\mathrm{n}) \\
(\mathrm{deg})\end{array}$ & $\begin{array}{c}\alpha \\
(\mathrm{deg})\end{array}$ & $\begin{array}{c}\gamma_{S L} \\
\left(\mathrm{~J} / \mathrm{m}^{2}\right)\end{array}$ & $\begin{array}{c}\gamma_{\alpha L}^{Z B} \\
\left(\mathrm{~J} / \mathrm{m}^{2}\right)\end{array}$ \\
\hline Value & 0.690 & 0.798 & 0.700 & 0.684 & 100 & 125 & -19.5 & 54.7 & 54.7 & 0.593 & 0.566 \\
$\left(\mathrm{~J} / \mathrm{m}^{2}\right)$ & $\left(\mathrm{J} / \mathrm{m}^{2}\right)$ & $\begin{array}{c}\gamma_{G a} \\
\left(\mathrm{~J} / \mathrm{m}^{2}\right)\end{array}$ & & & & \\
\hline Source & 21 & 20 & 20 & 22,28 & Exp. & Exp. & Exp. & Exp. & Exp. & Fit & Fit \\
\hline
\end{tabular}

\section{AUTHOR INFORMATION}

\section{Corresponding Author}

*E-mail: federico.panciera@c2n.upsaclay.fr (F.P.)

*E-mail: mirsaidov@nus.edu.sg (U.M.)

Author contribution: F.P., G.P., and J.-C.H. designed and conducted the experiments. Z.B., F.P., and U.M. developed the image analysis scripts and analyzed the data. V.G.D., F.P. and F.G. developed the model. J.-C.H. and L.T. developed the MBE sources. All authors have given approval to the final version of the manuscript.

\section{Funding Sources}

We acknowledge the ANR (French National Research Agency) for funding the NanoMAX ETEM through the TEMPOS grant, project number 10-EQPX-0050 and the Singapore National Research Foundation's Competitive Research Program funding (NRF-CRP16-2015-05). VGD thanks the Russian Science Foundation for financial support under the Grant 19-72-30004. 


\section{ACKNOWLEDGMENT}

We acknowledge Jean-Luc Maurice and Odile Stephane, directors of NanoMAX and TEMPOS, respectively, for their continuous support, and Ileana Florea for technical assistance.

\section{REFERENCES}

[1] Koguchi, M., Kakibayashi, H., Yazawa, M., Hiruma, K., Katsuyama, T., Crystal structure change of GaAs and InAs whiskers from zinc-blende to wurtzite type. Japanese journal of applied physics. 1992, 31(7R), 2061.

[2] Algra, R.E., Verheijen, M.A., Borgström, M.T., Feiner, L.F., Immink, G., van Enckevort, W.J., Vlieg, E. and Bakkers, E.P., Twinning superlattices in indium phosphide nanowires. Nature, 2008, 456(7220), 369.

[3] Caroff, P., Dick, K.A., Johansson, J., Messing, M.E., Deppert, K., Samuelson, L., Controlled polytypic and twin-plane superlattices in III-V nanowires. Nature Nanotechnology, 2009 4(1), 50.

[4] Persson, A.I., Larsson, M.W., Stenström, S., Ohlsson, B.J., Samuelson, L. and Wallenberg, L.R., Solid-phase diffusion mechanism for GaAs nanowire growth. Nature materials, 3(10), 677 (2004).

[5] Murayama, M. and Nakayama, T., Chemical trend of band offsets at wurtzite/zinc-blende heterocrystalline semiconductor interfaces. Physical Review B, 49(7), 4710 (1994).

[6] Spirkoska, D., Arbiol, J., Gustafsson, A., Conesa-Boj, S., Glas, F., Zardo, I., Heigoldt, M., Gass, M.H., Bleloch, A.L., Estrade, S., Kaniber, M., Structural and optical properties of high quality zinc-blende/wurtzite GaAs nanowire heterostructures. Physical Review B, 80(24), 245325 (2009).

[7] Akopian, N., Patriarche, G., Liu, L., Harmand, J.C., Zwiller, V., Crystal phase quantum dots. Nano letters, 10(4), 1198-1201 (2010).

[8] Taherkhani, M., Willatzen, M., Mørk, J., Gregersen, N., McCutcheon, D.P., Type-II quantum-dot-in-nanowire structures with large oscillator strength for optical quantum gate applications. Physical Review B, 96(12), 125408 (2017). 
[9] Loitsch, B., Winnerl, J., Grimaldi, G., Wierzbowski, J., Rudolph, D., Morkötter, S., Döblinger, M., Abstreiter, G., Koblmüller, G., Finley, J.J., Crystal phase quantum dots in the ultrathin core of GaAs-AlGaAs core-shell nanowires. Nano Letters, 15(11), 7544-7551 (2015). [10] Assali, S., Lähnemann, J., Vu, T. T. T., Jöns, K. D., Gagliano, L., Verheijen, M. A., Akopian, N., Bakkers, E.P., Haverkort, J.E., Crystal phase quantum well emission with digital control. Nano Letters, 17(10), 6062-6068 (2017).

[11] Glas, F., Harmand, J.C., Patriarche, G., Why does wurtzite form in nanowires of III-V zinc blende semiconductors? Physical Review Letters, 99(14), 146101 (2007).

[12] Jacobsson, D., Panciera, F., Tersoff, J., Reuter, M.C., Lehmann, S., Hofmann, S., Dick, K.A., Ross, F.M., Interface dynamics and crystal phase switching in GaAs nanowires. Nature, 531(7594), 317 (2016).

[13] Dastjerdi, M. H. T., Boulanger, J. P., Kuyanov, P., Aagesen, M. and LaPierre, R. R., Methods of Ga droplet consumption for improved GaAs nanowire solar cell efficiency. Nanotechnology, 27(47), 475403 (2016).

[14] Panciera, F., Norton, M. M., Alam, S. B., Hofmann, S., Mølhave, K., Ross, F. M., Controlling nanowire growth through electric field-induced deformation of the catalyst droplet. Nature Communications 7, 12271 (2016).

[15] Harmand, J. C., Patriarche, G., Glas, F., Panciera, F., Florea, I., Maurice, J. L., Travers, L., Ollivier, Y., Atomic step flow on a nanofacet. Physical Review Letters, 121(16), 166101 (2018). [16] Schwarz, K. W., Tersoff, J. Elementary processes in nanowire growth. Nano letters, 11(2), 316-320 (2010).

[17] Schwarz, K. W., Tersoff, J., Kodambaka, S., Chou, Y. C., Ross, F. M. Geometrical frustration in nanowire growth. Physical Review Letters, 107(26), 265502 (2011).

[18] Krogstrup, P., Popovitz-Biro, R., Johnson, E., Madsen, M. H., Nygård, J., \& Shtrikman, H. Structural phase control in self-catalyzed growth of GaAs nanowires on silicon (111). Nano Letters, 10(11), 4475-4482 (2010).

[19] Dubrovskii, V. G. Development of growth theory for vapor-liquid-solid nanowires: contact angle, truncated facets, and crystal phase. Crystal Growth \& Design 17(5), 2544-2548 (2017). [20] Pankoke, V., Kratzer, P., Sakong, S., Calculation of the diameter-dependent polytypism in GaAs nanowires from an atomic motif expansion of the formation energy. Physical Review B, 84, 075455 (2011). 
[21] Moll, N., Kley, A., Pehlke, E., Scheffler, M., GaAs equilibrium crystal shape from first principles, Physical Review B, 54, 8844 (1996).

[22] Dubrovskii, V. G., Sibirev, N. V., Cirlin, G. E.; Soshnikov, I. P., Chen, W. H., Larde, R., Cadel, E., Pareige, P., Xu, T., Grandidier, B., Nys, J.-P., Stievenard, D., Moewe, M., Chuang, L. C., Chang-Hasnain, C. Gibbs-Thomson and diffusion-induced contributions to the growth rate of Si, InP and GaAs nanowires, Physical Review B 79, 205316 (2009).

[23] Rudolph, D., Hertenberger, S., Bolte, S., Paosangthong, W., Spirkoska, D., Doblinger, M., Bichler, M., Finley, J. J., Abstreiter, G., Koblmuller, G. Nano Letters 11, 3848 (2011).

[24] Dubrovskii, V. G., Cirlin, G. E., Sibirev, N. V., Jabeen, F., Harmand, J. C., Werner, P. New mode of vapor-liquid-solid nanowire growth, Nano Letters 11, 1247-1253 (2011).

[25] Plissard, S., Dick, K.A., Larrieu, G., Godey, S., Addad, A., Wallart, X. and Caroff, P., Goldfree growth of GaAs nanowires on silicon: arrays and polytypism. Nanotechnology, 21(38), 385602 (2010).

[26] Lehmann, S., Jacobsson, D., Dick, K.A., Crystal phase control in GaAs nanowires: opposing trends in the Ga-and As-limited growth regimes. Nanotechnology, 2015 26(30), 301001.

[27] Kim, W., Dubrovskii, V. G., Vukajlovic-Plestina, J., Tütüncüoglu, G., Francaviglia, L., Güniat, L., Potts, H., Friedl, M., Leran, J.-P., Fontcuberta i Morral, A. Bi-stability of contact angle and its role in achieving quantum-thin self-assisted GaAs nanowires. Nano Letters, 2018 $18,49-57$.

[28] Hardy, S.C., The surface tension of liquid gallium. Journal of Crystal Growth, 1985, 71(3), 602-606. 


\section{Supporting Information}

\section{Table of Materials}

S1. Image processing and data extraction

S2. Calculation of Ga and As fluxes from kinetic data

S2.1 Calculation of Ga flux from droplet growth rate

S2.2 Calculation of As flux from nanowire axial growth rate

S3. Variability of critical contact angles

S4 Details of the model

S5 Supplementary Figures

S6 Supplementary Movie caption

S7 Supplementary references

\section{S1. Image processing and data extraction}

The image processing algorithm for the analysis of in situ image sequence file were written in Python-2.7 using scipy, ${ }^{1}$ numpy, ${ }^{2}$ scikit-image, ${ }^{3}$ opencv, ${ }^{4}$ mpi4 py $^{5}$ and matplotlib ${ }^{6}$ libraries .

The image processing routine can be divided into two main parts: image segmentation and Hough transform applied to the nanodroplet and the nanowire. Both steps were done separately for identification of the droplet and nanowire boundaries, and the liquid-solid interface.

For image segmentation, the intensity-based thresholding algorithms are unsuitable for our datasets because the contrast around the droplet is non-uniform. Here, the background subtraction cannot effectively remove the contrast gradient because the object size is comparable to the background. To address this challenge in the segmentation, we employed the Canny edgedetection algorithm, ${ }^{7}$ a widely used gradient-based segmentation method, from the scikit-image library. As a preliminary image processing step, this function also applies the Gaussian blurring to remove the high-frequency noise. However, the choice of $\sigma$, the standard deviation of Gaussian function is different for the droplet-nanowire ( $\sigma=5$ pixels) and droplet boundary ( $\sigma=7-10$ pixels), which are low and high-frequency image features, respectively. Subsequently, in order to detect the edges, the Canny algorithm requires an input of low and high thresholds, which were chosen manually for each movie. Later these two sets of binary images were extensively used, and, for 
the sake of clarity, we refer to them as the droplet edges (Figure S1b) and interface edges (Figure S1c) throughout the text.

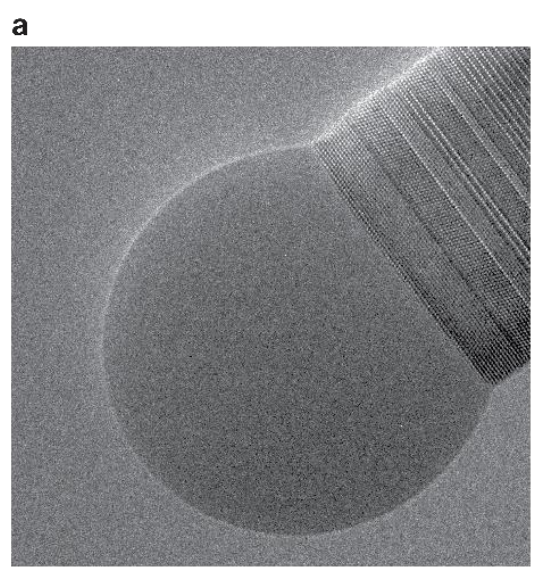

b

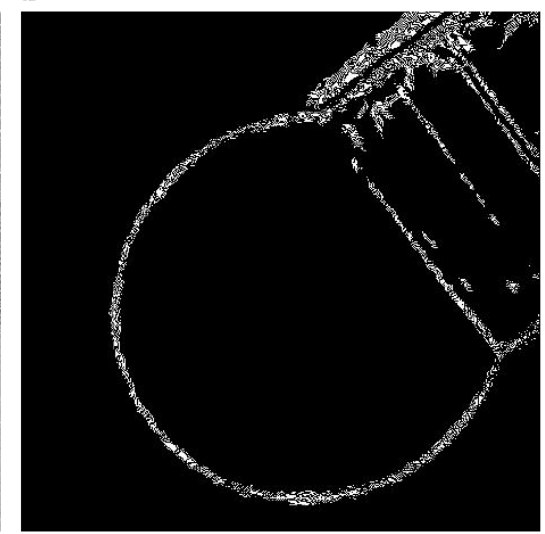

d

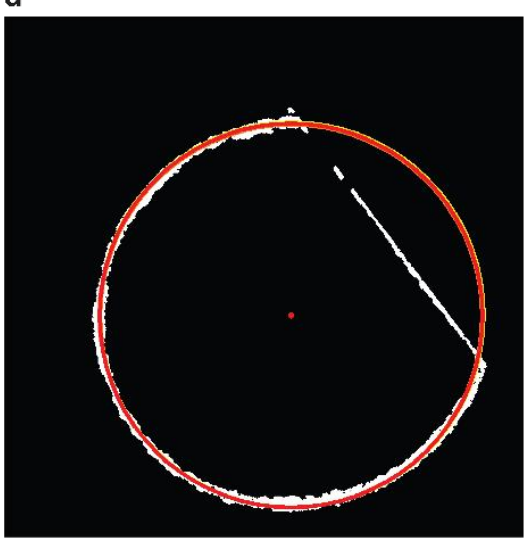

e

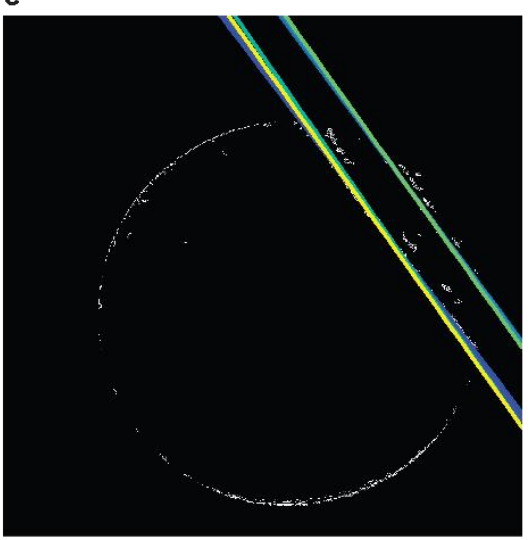

c

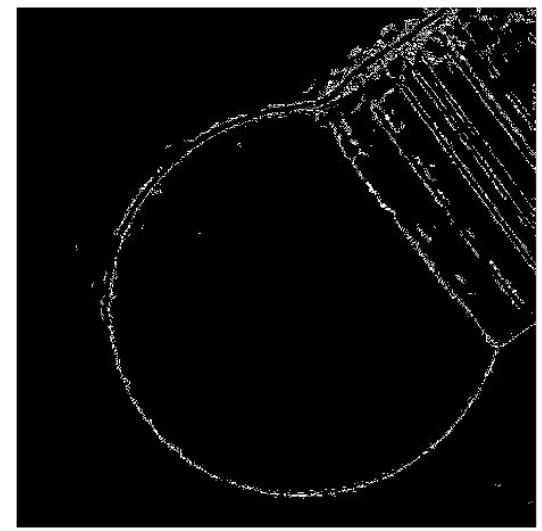

f

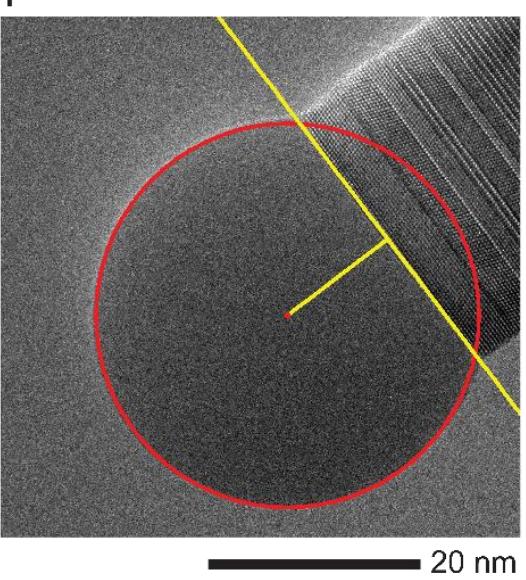

Figure S1 a. Raw grayscale image of the nanodroplet and nanowire. b. Droplet edges: Result of the Canny processing (thresholds - 7, 9 pixels), after the Gaussian blur with $\sigma=8$ pixels. c. Interface edges: Result of the Canny processing (thresholds -10, 12 pixels) after the Gaussian blur with $\sigma=5$ pixels. d. Hough circle transform on the masked "droplet edges" returns best fit at $\mathrm{R}=332$ pixels $(18.04 \mathrm{~nm})$ e. Masking the "interface edges" will highlight lines located at the interface, as shown by the Hough lines transform $\mathbf{f}$. Superimposed raw grayscale image with the best fit results of the Hough transform.

Once the edges of the droplet and the interface were detected, we executed the Hough transformations on these sequences in order to measure the dimensions of the droplet and nanowire during the growth. Note that the growth and orientation of the nanowire is perpendicular to the electron beam. Consequently, the projection of the spherical nanodroplet becomes a circular segment, whose center and radius can be measured using the Hough circles transform. ${ }^{8}$ The projection of the interface is a straight line, which was identified using the Hough lines transform. ${ }^{8}$ Since both the droplet and interface edges contain segmented objects from the entire field of view, 
it is necessary to perform masking in order to isolate the area of interest for each set. For this reason, the first execution of the Hough transforms delivers masks for the second iteration. Likewise, the Hough lines transform of the interface edge returns all the lines that are parallel to the interface and represent layers of the nanowire with the highest intensity gradient. Knowing this region, we can create a mask to separate the droplet from the nanowire and, hence, by applying this mask to the droplet edges, a more accurate result of the Hough circle transform is achieved (Fig S1d). Similarly, the result of the Hough circle transform on the droplet edges is used to create a circular mask, which eliminates the area outside of the droplet on the interface edges. Subsequently, we detect the interface with the second iteration of the Hough lines transform (Fig. S1e).

After the radius $r$ and positions of the droplet centre and the interface line have been identified, we measure the minimum distance $h$ from the centre to the interface, and find the total height $H$ of the droplet and its volume $V(r, H)$ for each frame using

$$
\begin{gathered}
H=h+r, \\
V=\frac{2 \pi H^{2}(3 r-H)}{3} .
\end{gathered}
$$

Another important dimension that characterizes the morphology is the nanowire diameter, given by the projected length of the droplet-nanowire interface. Note that in the case of large ZB NW sections, when the contact angle is above $125^{\circ}$, the tip of the nanowire is submerged into the droplet, so the measurement at the interface would overestimate the diameter of the growing front. Therefore, for every frame, we look at the two cross-sections: at the interface and at a fixed distance of 25 pixels $(1.36 \mathrm{~nm})$ away from the interface. For both cross-sections, we calculate the mean intensity within 5 pixels $(0.27 \mathrm{~nm})$, and by stacking these $1 \mathrm{D}$ arrays together for each movie, we create two sets of kymowires: the interface (Figure S2b) and shifted (Figure S2c). Segmentation of the two kymowires is similar. During the imaging, the lower corner of the nanowire is always kept at the focus, and the upper corner is kept under the focus. Therefore, in order to find the edges, we divided the kymowire into two halves. For the upper half, we search for the local maximum using the scipy package, while the lower half is segmented by setting a manual intensity threshold at 100. 

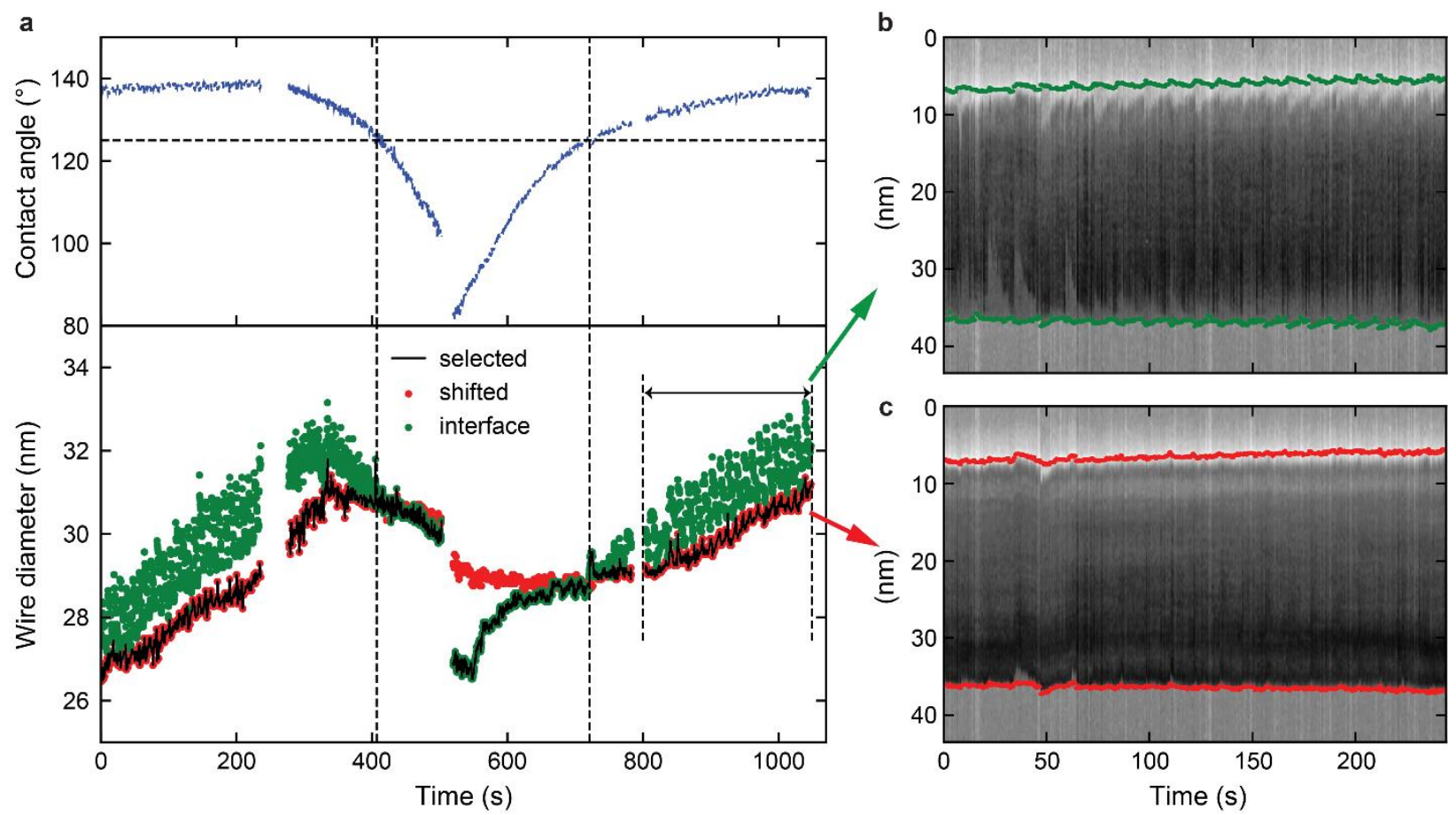

Figure S2. a. Contact angle (top) and nanowire diameter (bottom) versus time. Diameters are measured from the "shifted" (red circles) and "interface" (green circles) kymowires and the true diameter is selected (black solid curve) based on the contact angle value. Note how the two diameters are overlapping at the contact angles close to $125^{\circ}$. $\mathbf{b}$. The "interface" kymowire is obtained by stacking together the cross-sections at the interface. Boundaries of the kymowire are highlighted in green. For the ZB phase, the diameter measurements will be affected by the corner oscillations. c. The "Shifted" kymowire is similar to the "interface" kymowire, but the cross-sections are shifted away from the interface. Edges of the kymowire are shown in red. Measuring the diameter at the shifted interface ensures that the readings are insensitive to the corner oscillations. However, these measurements are unable to track the fast diameter changes at small contact angles.

Finally, after finding the diameters of both kymowires, we make the selection of the true nanowire diameter based on the value of the contact angle. For contact angles greater than $125^{\circ}$, the shifted diameter is selected, while for smaller angles, we measure the diameter at the interface.

\section{S2. Calculation of Ga and As fluxes from the kinetic data}

$\underline{\text { S2.1 Calculation of Ga flux from the droplet growth rate }}$

We modified the droplet volume by changing both Ga and As fluxes. During growth of the first 175 MLs, we kept the Ga flux constant and modulated the As flux. After that, we kept the As flux constant and periodically turned on and off the Ga flux. Figure 2a of the main text shows that the V/III flux ratio is the key parameter for controlling the droplet volume. While the droplet volume changes at different rates under different growth conditions, it consistently increases under an excess of $\mathrm{Ga}$ and decreases under an excess of As. A simple calculation (see below) shows that, 
in our conditions, the Ga diffusion along the nanowire sidewalls to the top is the main mechanism governing the droplet volume change.
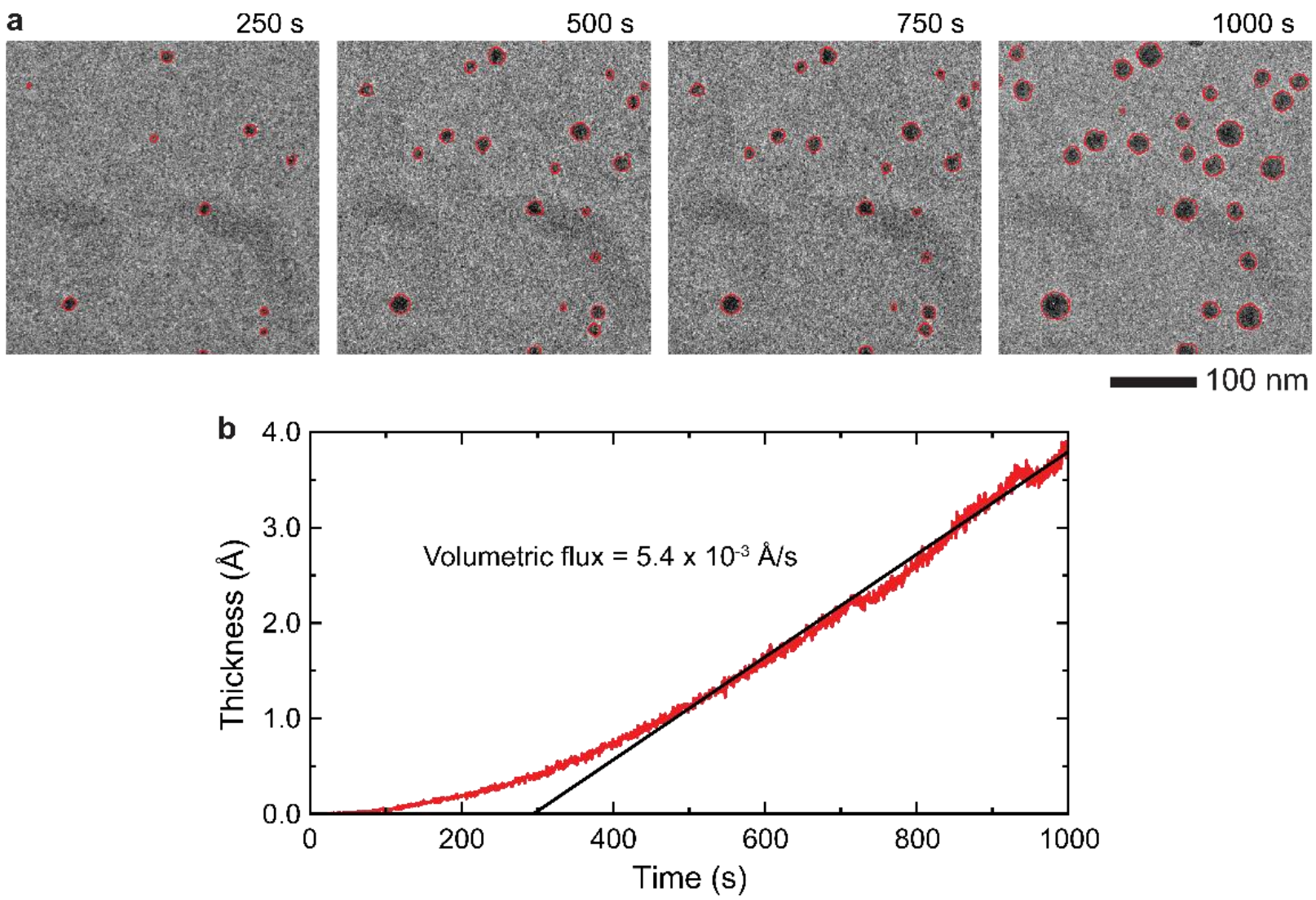

Figure S3. a. Evolution of the Ga droplet size and density with time. The nanodroplet boundaries are highlighted in red. b. Equivalent thickness of the deposited Ga versus time.

In order to calculate the Ga flux from the MBE source, we perform a special experiment, where $\mathrm{Ga}$ is deposited on a $\mathrm{SiN}_{\mathrm{x}}$ membrane, which is heated up to $420{ }^{\circ} \mathrm{C}$. Once the deposition starts, spherical Ga nanodroplets begin to nucleate and grow on the substrate surface (Fig. S3a). By segmenting the droplets and assuming hemispherical shape, we are able to calculate the total volume of $\mathrm{Ga}$ as a function of time. Experiments conducted at different temperatures allow us to conclude that desorption from the droplet surface and from the substrate is negligible for temperature below $500{ }^{\circ} \mathrm{C}$. Then the Ga volume per unit area exactly equals the Ga flux onto the surface (Figure S3b).

The increase of the droplet volume on the nanowire top can be due to the direct impingement of $\mathrm{Ga}$ atoms onto the droplet surface or their diffusion along the nanowire sidewalls, while the volume reduction can be due to evaporation, incorporation into the crystal phase or reverse 
diffusion. We can estimate the contribution of all these processes using the data shown in Figure 2. In the range from layer \#200 to \#240, the maximum rate of changing the droplet volume is $150 \mathrm{~nm}^{3} / \mathrm{s}$ (in both directions). The axial nanowire growth rate is $0.1 \mathrm{ML} / \mathrm{s}$, the nanowire diameter is $30 \mathrm{~nm}$, and the contact angle is $100^{\circ}$. First, we can exclude any contribution from evaporation as it is negligible at this temperature. ${ }^{9}$ The volume of Ga impinging onto the droplet from the direct flux is $<10 \mathrm{~nm}^{3} / \mathrm{s}$, while the volume of Ga consumed to grow the nanowire is $\sim 10 \mathrm{~nm}^{3} / \mathrm{s}$. Both contributions are not sufficient to explain the rate of volume change of $\sim 150 \mathrm{~nm}^{3} / \mathrm{s}$. This leads us to conclude that the main contribution to the droplet volume change originates from the $\mathrm{Ga}$ diffusion on the NW sidewalls.

\section{$\underline{\text { S2.2 Calculation of As flux from the axial growth rate }}$}

Figure S4 shows that the axial nanowire growth rate depends on the As pressure in the source as well as on the droplet size. Higher As flux at higher pressure yields a larger amount of As reaching the droplet surface, while larger droplet represents a larger collection area. In the Aslimited regime, as in our case, the instantaneous axial growth rate equals the As flux times the cross-sectional area of the droplet (provided that the As evaporation is negligible).

Let us consider cylindrical nanowire with spherical cap droplet of the radius $r$ and height $h$ seated on the top. The total flux of As entering the droplet, $\Phi$, equals the flux per unit area, $j$, times the cross-sectional area of the droplet, $S$, which gives $\Phi=j S$. The nanowire growth direction is parallel to the As flux, so for contact angles smaller than $90^{\circ}$ the cross-section equals exactly $S=$ $\pi r^{2}$. The volume of each new monolayer equals the (111) interlayer distance, $b=0.326 \mathrm{~nm}$, multiplied by the interface area, $A=\pi d^{2} / 4$. The time interval between the two successive nucleation events, $\tau$, equals the volume of the monolayer divided by the volume of material entering the droplet per unit time:

$$
\tau=\frac{b A}{\Phi}=\frac{b d^{2}}{4 j r h}
$$




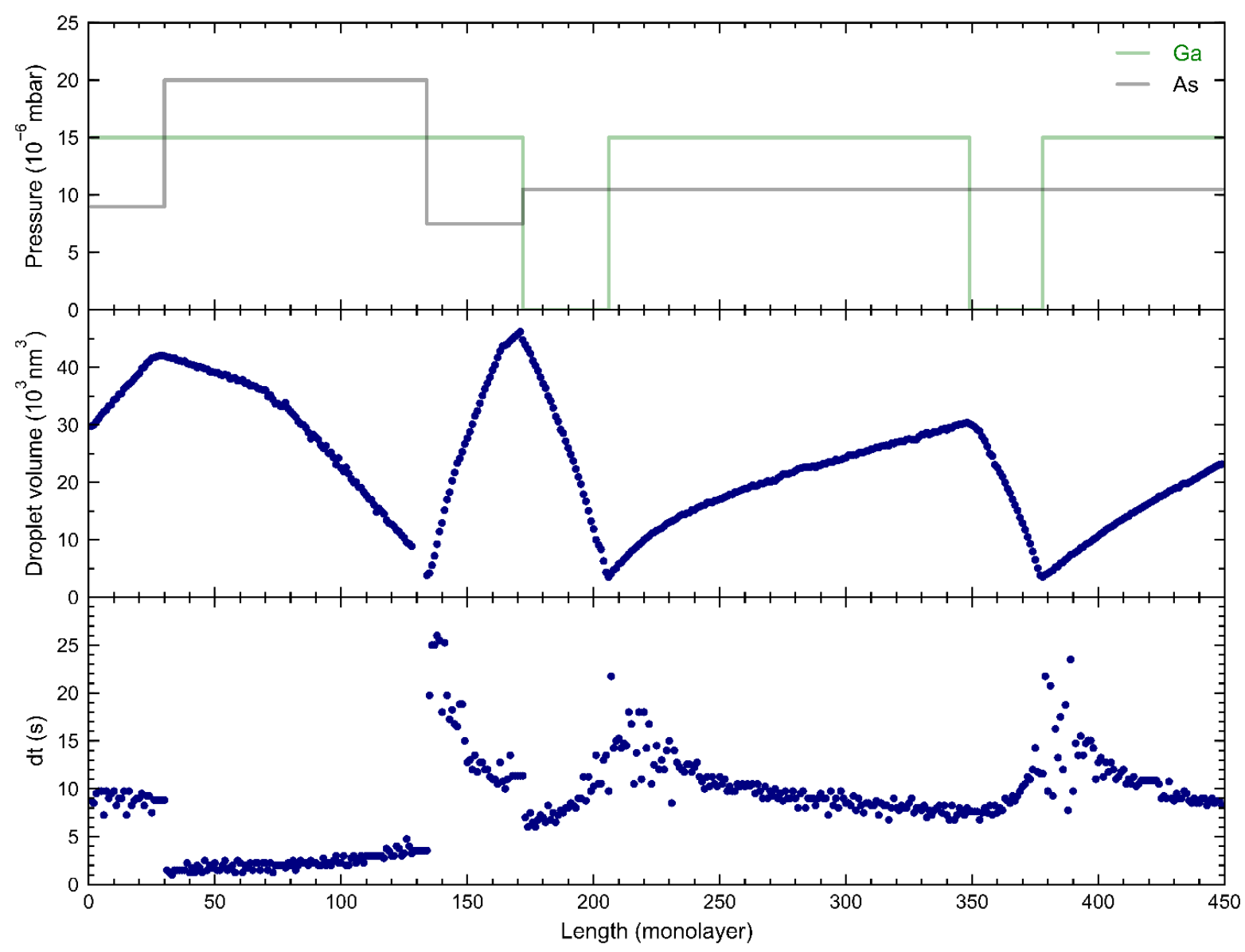

Figure S4. Fluxes of Ga and As (top), droplet volume (middle) and time interval between successive monolayer nucleation events (bottom), plotted as functions of the nanowire length expressed in monolayers.

We then plot $\tau$ as a function of the droplet geometrical parameter $d^{2} / r^{2}$ and fit linearly each data set measured for different values of the As pressure in the source (Figure S5). The fact that linear fits match very well the data supports our hypothesis of As being collected exclusively by the droplet surface. The slope of each line is inversely proportional to the As flux $j$. This allows us to extrapolate the As flux as a function of the As pressure in the source (see the inset in Figure S5). 


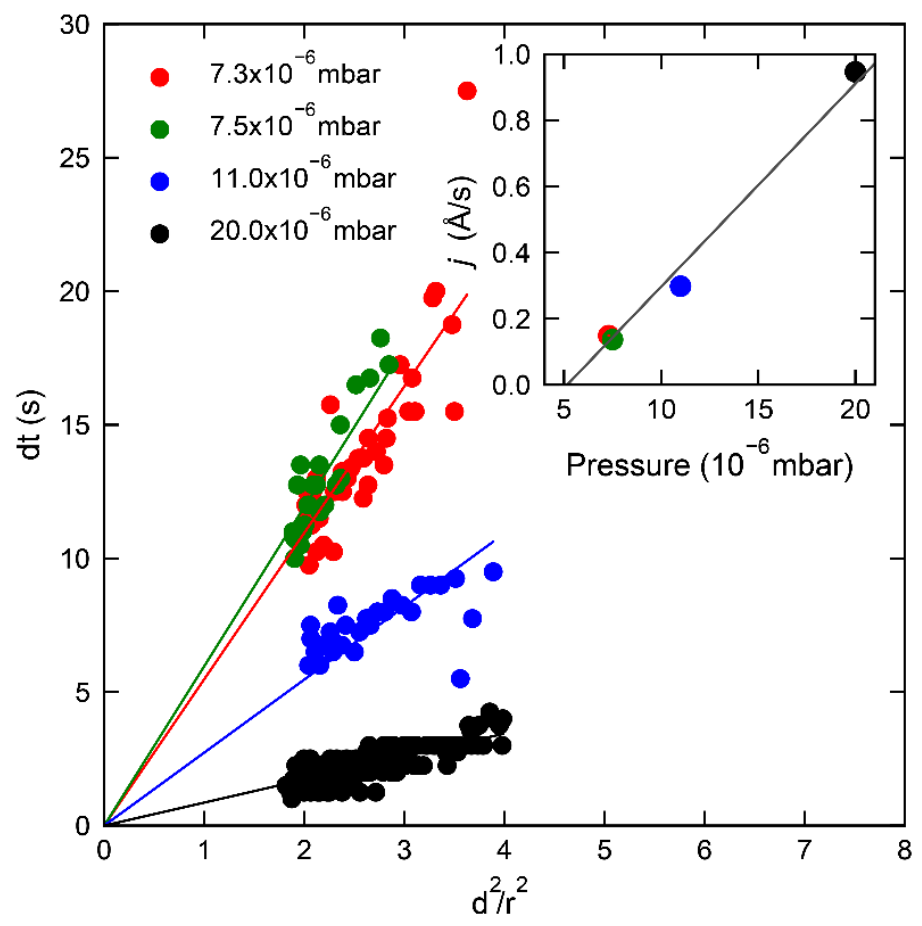

Figure S5. Nanowire axial growth rates plotted as functions of $d^{2} / r^{2}$ and extrapolated by linear fits. The slope of each line is inversely proportional to the As flux at a given As pressure in the source. The inset shows the obtained dependence of the As flux on the As pressure in the source, and its linear fit.

\section{S3. Variability of the critical contact angles}

Here, we systematically investigate the variability of the critical contact angles $\varphi_{\min }$ and $\varphi_{\max }$. We observed the phase switching in nanowires having diameters in the range from 15 $\mathrm{nm}$ to $65 \mathrm{~nm}$, growth rates from $0.05 \mathrm{ML} / \mathrm{s}$ to $1 \mathrm{ML} / \mathrm{s}$, and grown at temperatures between $350{ }^{\circ} \mathrm{C}$ and $480{ }^{\circ} \mathrm{C}$. The measured critical angles are plotted in Figure S6 and show no clear trend with these parameters. We speculate that the observed variability could be due to the different sidewall structure. Pure WZ or ZB nanowires have different stable sidewall facets that are rotated by $30^{\circ}$. When the phase changes, the nanowire sidewalls require a certain length to reconfigure. Therefore, the short WZ segments shown in Figure 2 may still maintain the sidewall orientation of the ZB phase or even have a more complex structure. On the other hand, the long WZ segment shown in Figure 3 should have the typical sidewall structure of the WZ nanowire. For this reason, the development of tapering and hence switching from the $\mathrm{ZB}$ to $\mathrm{WZ}$ phase can occur at different contact angles. 


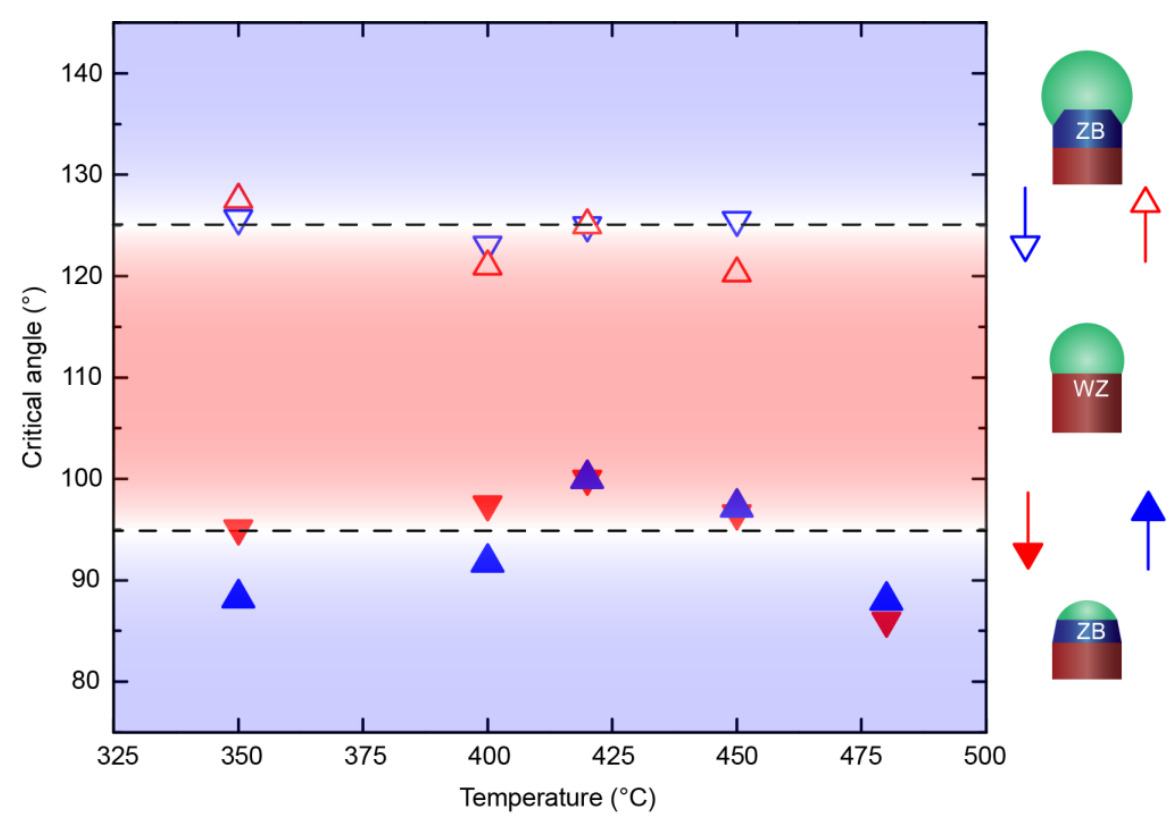

Figure S6. Critical angles for the phase transition at different temperatures. Each point corresponds to nucleation of the first monolayer of a different phase. Data at 350,400 and $450{ }^{\circ} \mathrm{C}$ are collected from a sequence of experiments on the same nanowire; data at $420{ }^{\circ} \mathrm{C}$ are the average of the measurements represented in Fig. 2; data at $480{ }^{\circ} \mathrm{C}$ are from Fig.3. Empty symbols represent the transition at large angles, while solid symbols represent the transition at small angles. Downward (upward) triangles indicate that the droplet is shrinking (swelling). Red (blue) colour represents the phase change from WZ to ZB (ZB to WZ). The critical angles seem to be independent of temperature in the investigated range.

\section{S4 Details of the model}

In modeling, we use Eq. (1) of the main text, assuming that inward tapering of NWs at small contact angles is preferred in the $\mathrm{ZB}$ phase, that is, the (110) ZB inclined facet is the lowest energy one. The curves in Fig. 4 of the main text are obtained for the same (110) facet for tapered and truncated NWs, making an angle of $54.7^{\circ}$ to the vertical. The two crossing points at the minimum and maximum contact angles are fitted by adjusting the two surface energies of the liquid-solid interfaces, as explained in the main text. Figure $\mathrm{S} 7$ shows how the curves change at a fixed $\varphi_{\max }=$ $125^{\circ}$ and $\varphi_{\min }$ decreasing from $100^{\circ}$ to $90^{\circ}$. The fitting values of the solid-liquid surface energies $\gamma_{S L}$ and $\gamma_{\alpha L}$ are $0.540 \mathrm{~J} / \mathrm{m}^{2}$ and $0.522 \mathrm{~J} / \mathrm{m}^{2}$ at $\varphi_{\min }=95^{\circ}$, and $0.477 \mathrm{~J} / \mathrm{m}^{2}$ and $0.471 \mathrm{~J} / \mathrm{m}^{2}$ at $\varphi_{\min }=$ $90^{\circ}$. It is seen that decreasing the $\varphi_{\min }$ suppresses the possibility of forming vertical GaAs NWs in ZB phase at a fixed growth temperature of $420{ }^{\circ} \mathrm{C}$. 


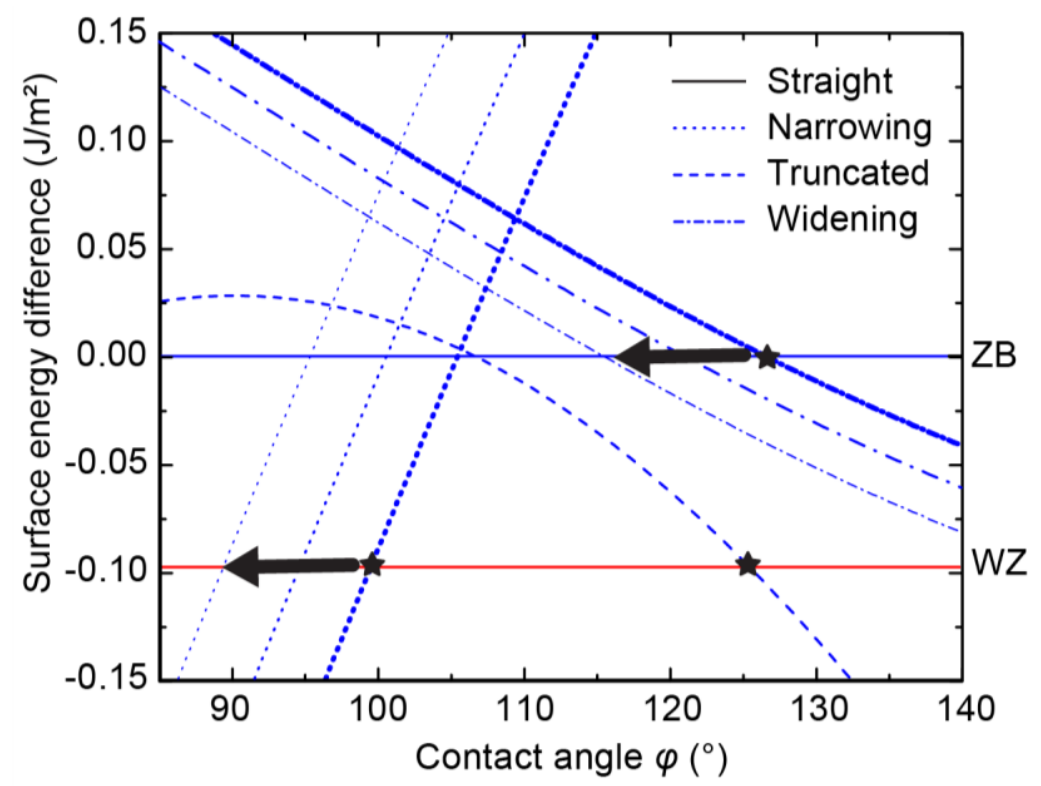

Figure S7. Surface energy curves for different $\varphi_{\min }$ from $\mathbf{9 0}^{\circ}$ to $\mathbf{1 0 0}^{\circ}$. The arrows show modifications of the lines as $\varphi_{\min }$ decreases, which closes the region of vertical ZB NWs at large contact angles.

It should be noted that these considerations are based entirely on the surface energetics of ZB or WZ NWs in different morphologies and ignore the influence of chemical potential on the crystal phase switching (see Ref. 11 of the main text). In this simplified picture, the preferred crystal phase is controlled by the sole parameter, the droplet contact angle, which is supported by the data. While including the chemical potential considerations may refine the picture, the existence of the two stable critical contact angles for the crystal phase switching in GaAs NWs (which should be very similar for gold- and gallium-catalyzed growths) strongly suggests that phase transitions occur quite abruptly whenever allowed by surface energetics. In other words, chemical potential near the transition points quickly exceeds the energy of stacking fault, which is required for switching from $\mathrm{ZB}$ to $\mathrm{WZ}$ phase, ${ }^{11}$ and do not significantly affect the critical contact angles for the transition. 


\section{S5 Supplementary Figures}
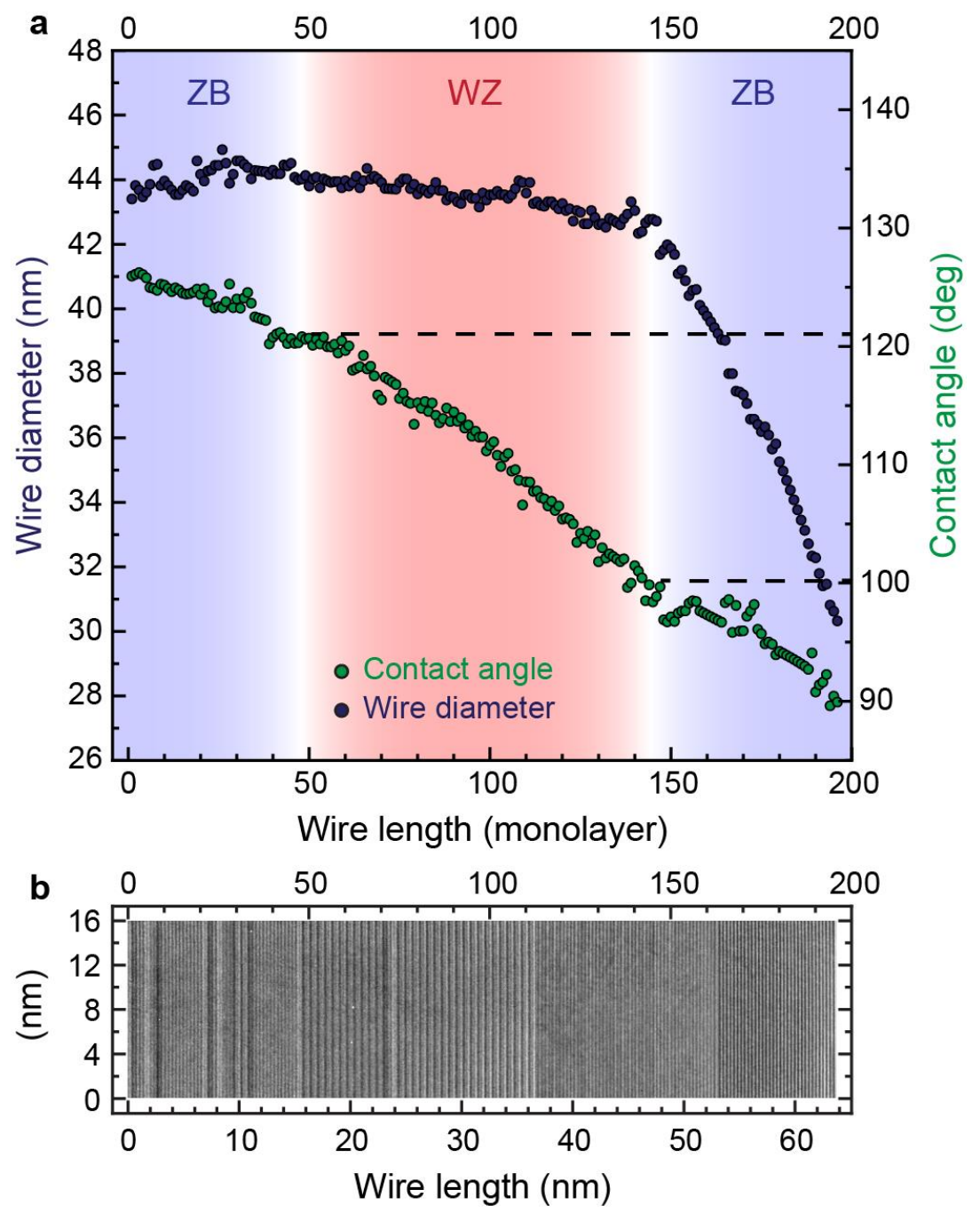

Figure S8. Evolution of GaSb nanowire morphology with time. a. Diameter and contact angle plotted as functions of the nanowire length. The two critical angles are $120^{\circ}$ and $95^{\circ}$, and the transition to $\mathrm{ZB}$ at small contact angle occurs simultaneously with the nanowire tapering. $\mathbf{b}$. Image of a slice of the observed nanowire reconstructed by composing 3 TEM images. 

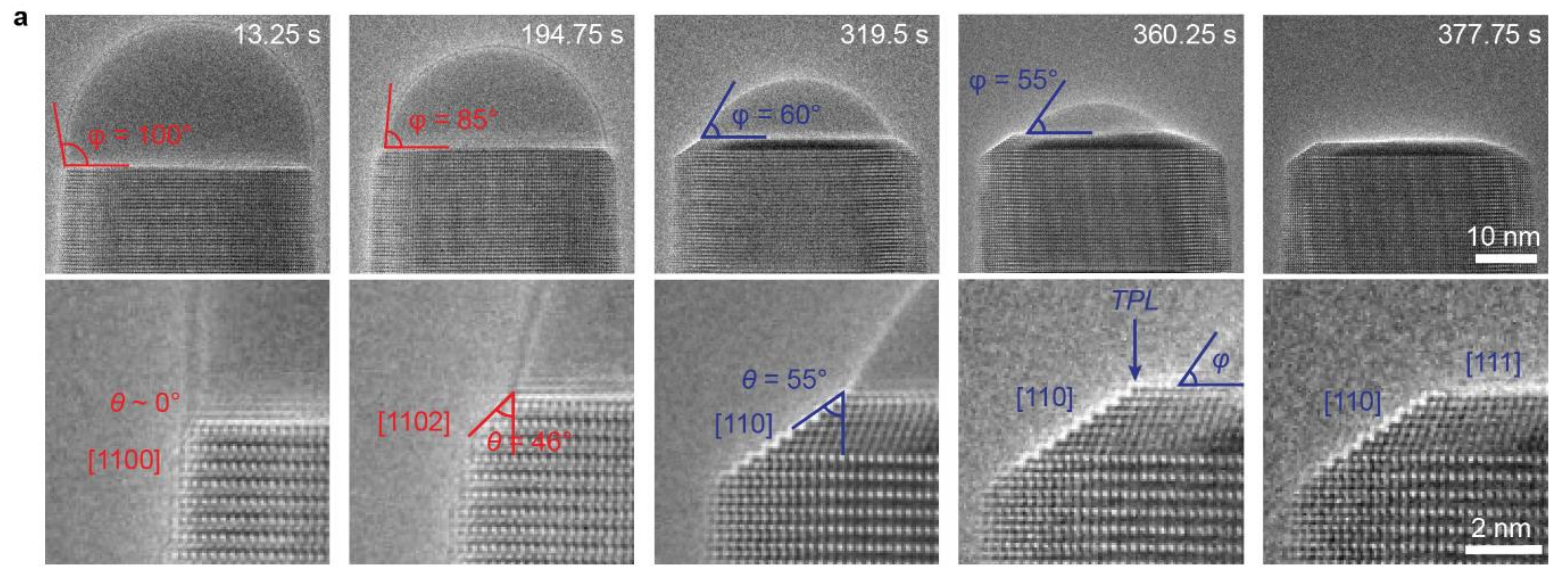

b

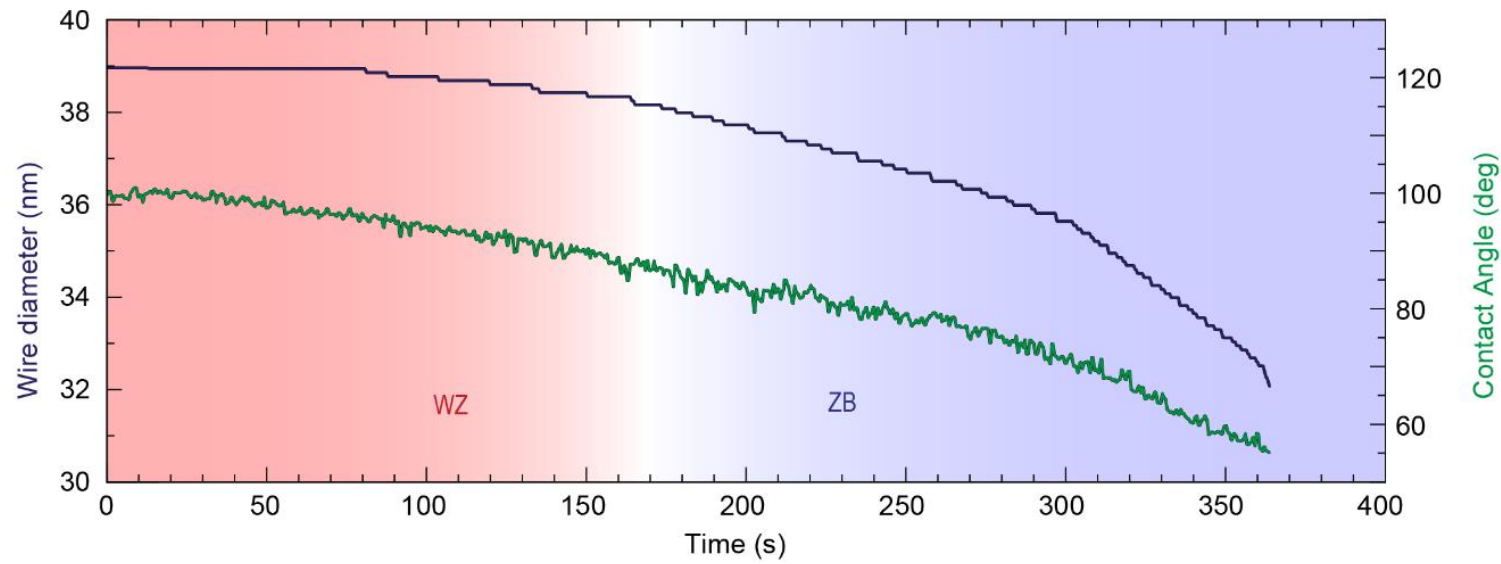

Figure S9. Phase change at the small contact angle. a. Sequence of images showing the switch from $\mathrm{WZ}$ to $\mathrm{ZB}$ at the small contact angle and the corresponding details of the TPL with the taper angle $\theta$ and finally depinning of the droplet. The tapering starts in the WZ phase, and the phase switching to ZB occurs successively. When the contact angle reaches a critical value of $\sim 55^{\circ}$, the droplet unpins from the TPL and moves on the $\{111\}$ surface until the full consumption. b. Graphs showing the nanowire diameter and the contact angle versus time until the droplet unpinning from the TPL.

\section{S6 Supplementary Movie caption}

- Movie 1. Growth of ZB at large contact angle $\left(\varphi>125^{\circ}\right)$. Recorded at a frame rate of 4 fps, decimated by 2 and played at $12 \mathrm{fps}$.

- Movie 2. Growth of WZ at intermediate contact angle $\left(125^{\circ}>\varphi>100^{\circ}\right)$. Recorded at a frame rate of $4 \mathrm{fps}$, decimated by 2 and played at $12 \mathrm{fps}$.

- Movie 3. Growth of ZB at small contact angle $\left(\varphi<100^{\circ}\right)$. Recorded at a frame rate of 4 fps, decimated by 2 and played at $12 \mathrm{fps}$.

- Movie 4. Transition from WZ to ZB and ZB to WZ at small contact angle. Recorded at 4 fps, decimated by 10 and played at $10 \mathrm{fps}$. 


\section{S7 Supplementary references}

1. Oliphant, T.E. Python for Scientific Computing. Computing in Science Engineering 9, 10-20 (2007).

2. Walt, S. van der, van der Walt, S., Chris Colbert, S. \& Varoquaux, G. The NumPy Array: A Structure for Efficient Numerical Computation. Computing in Science \& Engineering 13, 22-30 (2011).

3. Walt, S. van der et al. scikit-image: image processing in Python. PeerJ 2, e453 (2014).

4. Bradski, G. \& Kaehler, A. Learning OpenCV: Computer Vision with the OpenCV Library. ('O'Reilly Media, Inc.', 2008).

5. Gropp, W.,. Gropp, W. D., Argonne Distinguished Fellow Emeritus, Lusk, E. \& Skjellum, A. Using MPI: Portable Parallel Programming with the Message-passing Interface. (MIT Press, 1999).

6. Hunter, J. D. Matplotlib: A 2D Graphics Environment. Comput. Sci. Eng. 9, 90-95 (2007).

7. Canny, J. A Computational Approach to Edge Detection. IEEE Transactions on Pattern Analysis and Machine Intelligence PAMI-8, 679-698 (1986).

8. Duda, R. O. \& Hart, P. E. Use of the Hough transformation to detect lines and curves in pictures. Communications of the ACM 15, 11-15 (1972).

9. Glas, F., Ramdani, M. R., Patriarche, G. \& Harmand, J.-C. Predictive modeling of selfcatalyzed III-V nanowire growth. Phys. Rev. B Condens. Matter 88, 195304 (2013). 